\title{
Nonsense-mediated mRNA decay and development: shoot the messenger to survive?
}

\author{
Marta Vicente-Crespo* and Isabel M. Palacios ${ }^{\dagger}, 1$ \\ 'Division of Biology, University of California San Diego, 9500 Gilman Drive, Bonner Hall 3230, La \\ Jolla, CA 92093-0322, U.S.A. \\ †Zoology Department, University of Cambridge, Downing Street, Cambridge CB2 3EJ, U.K.
}

\begin{abstract}
NMD (nonsense-mediated mRNA decay) is a surveillance mechanism that degrades transcripts containing nonsense mutations, preventing the translation of potentially harmful truncated proteins. Although the mechanistic details of NMD are gradually being understood, the physiological role of this RNA surveillance pathway still remains largely unknown. The core NMD genes $U p f 1$ (up-frameshift suppressor 1) and $U p f 2$ are essential for animal viability in the fruitfly, mouse and zebrafish. These findings may reflect an important role for NMD during animal development. Alternatively, the lethal phenotypes of upf1 and upf 2 mutants might be due to their function in NMD-independent processes. In the present paper, we describe the phenotypes observed when the NMD factors are mutated in various organisms, and discuss findings that might shed light on the function of NMD in cellular growth and development of an organism.
\end{abstract}

\section{Keywords}

gene expression; nonsense-mediated mRNA decay (NMD); premature termination codon (PTC); RNA decay; RNA processing; RNA quality control

\section{Introduction}

Severo Ochoa won the 1959 Nobel Prize in Medicine after he discovered how RNA is synthesized. The importance of RNA synthesis has been understood for many decades and, historically, the study of gene expression regulation has tended to concentrate on the means by which genes are switched on and RNAs are synthesized. However, there is another aspect of gene expression regulation that is arguably as important as RNA synthesis, and this is RNA turnover. It is well established that one mechanism of RNA degradation, NMD (nonsense-mediated mRNA decay), contributes to the rapid degradation of many mRNAs. Although the mechanistic details of NMD are gradually being understood, the physiological role of NMD still remains largely unknown. In the present paper, we review what is known about the role of this RNA surveillance pathway in cellular growth and organism development.

NMD is a conserved mRNA surveillance pathway that eliminates aberrant transcripts containing a PTC (premature termination codon) that can arise accidentally through various means, including gene mutation, aberrant mRNA splicing and directed gene rearrangements

\footnotetext{
(CThe Authors Journal compilation@2010 Biochemical Society

${ }^{1}$ To whom correspondence should be addressed (mip22@cam.ac.uk). .
} 
[1-4]. In addition to its quality control function, NMD also regulates the expression levels of, among others, mRNAs with uORFs (upstream open reading frames) and alternatively spliced mRNAs [5]. Genome-wide transcriptome-profiling analyses in various organisms have revealed that the expression levels of $3-10 \%$ of all mRNAs are affected by NMD (reviewed in [6]), and that one-third of alternative splicing events in humans have the potential to generate PTC-containing mRNAs [7-9].

The biological importance of NMD is highlighted by the fact that up to $30 \%$ of all mutations causing human disease generate mRNAs with a PTC [10]. In some instances, proteins encoded by nonsense mRNAs are functional, but they are not produced in sufficient amounts due to degradation via NMD. This is the case in aniridia, cystic fibrosis and Duchenne muscular dystrophy [9,11-13]. There are also cases in which NMD ameliorates the effects of PTC mutations. For example, patients with a PTC-containing $\beta$-globin allele that does not trigger NMD show ineffective erythropoiesis, whereas those with an NMDinducing PTC are phenotypically normal. Similar cases have been described for the reduction of dominant truncated forms of the tumour-suppressor proteins BRCA1 (breast cancer early-onset 1) and WT1 (Wilms' tumour 1) (reviewed in [14]). In addition, inhibition of NMD leads to a significant reduction in tumour growth [15].

The key effectors of NMD include the Upf (up-frameshift suppressor) proteins (Upf1, Upf2 and Upf3), and the Smg (suppressor with morphological effect on genitalia) proteins (Smg1 and Smg5-Smg9) (reviewed in [6]). Upf proteins are conserved from yeast to humans; the Smgs have orthologues in multicellular organisms, but not in yeast (Table 1). An exception, Smg7, is not conserved in Drosophila. Upf1 is an ATP-dependent helicase and RNAdependent ATPase, whose activity in human cells is stimulated by the binding of Upf2 and Upf3 [16]. Transcripts carrying PTCs are promptly degraded upon contact with molecular complexes that include Upf1, Upf2 and Upf3, but the detailed mechanisms of this process are not fully understood (Figure 1). Upf1 activity is regulated via cycles of phosphorylation and dephosphorylation, which depend on the activity of the kinase Smg1, but also require the additional Smg proteins [17-20]. Degradation is finally achieved either by the activity of the endonuclease Smg6, and/or via Smg5/Smg7 which provide a link to general cellular mRNA decay enzymes [21-23].

For the human disorder cases described above, disease-modulating clinical strategies to manipulate surveillance levels are under consideration. A prerequisite to the implementation of these approaches is to have full understanding of the normal biological roles of NMD factors in vivo, especially since some of the core components of the NMD machinery participate in cellular processes that are NMD-independent. In the present review, we discuss the role of these factors in development, as well as whether their impact on cellular growth and organism viability is due to NMD or to other functions. This review is based on published data as well as our recent observations in Drosophila.

\section{Function of NMD in organism development}

The importance of NMD effectors in cell viability and organism development varies across species (Table 1). The NMD pathway has been found in all eukaryote organisms that have been examined, but the repertoire of factors that mediate NMD is larger in higher than in lower eukaryotes. Most of the work in lower eukaryotes has been done with the budding yeast Saccharomyces cerevisiae. In this organism, the loss of the Upf proteins has no obvious effect on growth, although PTC-containing mRNAs are stabilized in mutants for upf1-upf3 [24-27]. The fact that UPF1-UPF3 are not essential genes in yeast suggests that the regulation of targets by NMD in this organism is not required for growth. Similar to the budding yeast, upf2-deleted Schizosaccharomyces pombe strains are viable and show no 
apparent growth abnormality $[28,29]$, whereas deletion of Upf1 produces abnormally long cells probably due to problems with cell-cycle progression. However, it is not yet clear whether this phenotype is due to lack of NMD or to some other function of the protein (S. De and S. Brogna, personal communication).

Genetic screens in the nematode Caenorhabditis elegans identified seven genes (SMG1$S M G 7$ ) that are required for the degradation of nonsense mutant mRNAs of various genes (see [30] and references therein). Inactivation of any of these NMD factors in C. elegans leads to viable nematode worms that present mild morphological effects on the genitalia. The abnormalities are more obvious in the male bursa than in the hermaphrodite vulva; both are swollen, although the anatomical components of the copulatory organs are all present and partly functional [31]. Thus the NMD pathway in the nematode is apparently dispensable for viability, although necessary for the correct morphogenesis of the reproductory structures.

Two novel NMD factors that are highly conserved have been identified in the nematode, Smgl1 and Smgl2 (Table 1) [30]. Interestingly, these two factors are essential for viability in $C$. elegans, which suggests that they may be involved in additional cellular functions.

In contrast with the nematode, cultured Drosophila $\mathrm{S} 2$ cells depleted of UPF1 and UPF2 arrest at $\mathrm{G}_{2} / \mathrm{M}$-phase, and an increase in the proportion of $\mathrm{G}_{2} / \mathrm{M}$-phase cells is also observed after depletion of UPF3, SMG5 and SMG6 [32]. Concordantly, upf1 and upf2 mutant clones induced in an otherwise heterozygous fruitfly show growth defects and mutant cells seem to die by apoptosis ([33] and P. Avery, M. Vicente-Crespo and I.M. Palacios, unpublished work). At the developmental level, upf 1 and upf 2 mutants survive through larval stages due to maternal contribution, since the germline provision of Upf1 and Upf2 is essential for embryonic development and patterning of the follicular epithelium. None of the cellular growth and developmental defects observed in upf 1 and upf 2 mutants are seen in upf 3 or smg1 mutant fruitflies, although upf3 mutants develop slightly slower than the wild-type ([33] and P. Avery, M. Vicente-Crespo and I.M. Palacios, unpublished work). The increase observed in $\mathrm{G}_{2} / \mathrm{M}$-phase cells when UPF3 is depleted in cultured cells could explain the slower development observed in upf3 mutants. There are yet no mutant studies of the other NMD effectors in Drosophila, although all Upf and Smg proteins have been shown to play a role in NMD in S2 cells [32].

It is interesting to note that the Drosophila EJC (exon junction complex) is required for the localization of oskar mRNA to the posterior pole of the oocyte, an event that is essential in the establishment of the embryonic body axes, and in the development of the germ cells [34]. However, in contrast with the function of the mammalian EJC in NMD, this complex does not function with the Upf proteins in RNA localization, since upf1, upf2 and upf3 mutant oocytes show no obvious defects in the distribution of the transcript (K. Gold, M. Vicente-Crespo and I.M. Palacios, unpublished work).

Many of the phenotypes described in Drosophila have been observed in vertebrates. In the case of zebrafish, knockdown of UPF1 and UPF2 causes embryonic lethality, whereas depletion of UPF3 and $S M G 1$ has little to no effect on viability [35]. Similar to UPF1 and UPF2, SMG5-SMG7 are also essential during embryogenesis. Embryos injected with Upf1 morpholinos exhibit extensive necrosis in the central nervous system, impaired eye development, abnormal somite morphogenesis and perturbation in the posterior axis elongation and in the yolk sac extension. Brain patterning is disturbed particularly at the midbrain-hindbrain boundary, which is greatly diminished. Similar to the Upf1 knockdown, an 80-90\% reduction of viability is observed in embryos depleted of Upf2 or Smg5-Smg7. The phenotypes caused by Upf2, Smg5 and Smg6 depletions resemble that of Upf1-depleted 
embryos. In contrast, Smg7 morphants display phenotypes with specific features that are not observed in other cases. These embryos show an elongated hindbrain; the appearance of the midbrain-hindbrain boundary is altered, but the boundary is still established. Only the strong phenotype shows stacked somites comparable with the phenotypes observed in Upf1, Upf2, Smg5 and Smg6 morphants. The phenotypic similarities between Upf1, Upf2, Smg5 and Smg6 morphants suggest that the observed defects are likely to be due to the inhibition of NMD, whereas Smg7 may have acquired additional functions.

The existence of two paralogues of Upf3 (a and b, also known as Upf3 and Upf3x) in vertebrates has made the study of Upf3-knockout animals difficult. In zebrafish, the double knockdown of Upf3a and Upf3b shows phenotypes comparable with the weak phenotypes observed in upf $1^{-/-}$brains, but embryonic survival was not strongly compromised. Finally, no phenotype was observed in zebrafish embryos depleted of Smg1.

As yet, no phenotypic data for Upf3-knockout animals exists for the murine system, but, similar to zebrafish and Drosophila, Upf1/Rent1 and Upf2 knockouts in mice cause early embryonic lethality [36,37]. In the case of Upf1, mutant blastocysts showed potent induction of apoptosis, and were unable to generate stable embryonic stem cell lines. In conjunction with the observation that the Upf1/Rent1 mRNA is expressed in unfertilized oocytes, early embryos and cultured ES cells, these data suggest that cellular lethality may initiate in mutant embryos once the oocyte-derived Upf1/Rent1 is exhausted [36].

The loss of full-length Upf2 in the haemopoietic system leads to a strong reduction of all major lineages of the bone marrow (haemopoietic progenitors, granulocytes and B-cells) and, consistent with this, depletion of Upf2 was accompanied by severe anaemia [37]. The detailed phenotypic analysis of the bone marrow deletion of full-length Upf2 demonstrates a requirement for Up2 in the maintenance of all tested stem and progenitor subsets within the bone marrow. In contrast, no changes are observed when full-length Upf2 is deleted only in the myeloid lineage. Elimination of full-length Upf 2 during T-cell development results in thymic atrophy with reduced numbers of T-lymphocytes and increased numbers of preapoptotic cells. The analyses of the T-cell receptor $\beta(T c r b)$ locus shows that NMD is very active in T-cells since the inactivation of the pathway leads to high levels of PTC-containing $T c r b$ mRNAs (PTC+; generated by unproductive genomic rearrangements), otherwise undetectable in a wild-type background. Interestingly, resting T-cells that do not contain PTC $+T c r b$ mRNAs can survive when UPF2 is inactive. Therefore accumulation of PTC+ mRNAs after elimination of full-length Upf2 is unbearable for resting T-cells and probably one of the causes of the thymic atrophy.

Similar to the murine system, human Upf1 is required for S-phase progression in cultured cells [38]. However, in contrast with the mouse, the reduction of Upf2 levels in human tissue culture has no effect on cellular proliferation. Although this result suggests that the function of Upf2 in cell division is not conserved in humans, it is important to note that the depletion of Upf2 in the human cells experiment was only partial, and that the residual Upf2 might support its function in cell growth.

The only data available in humans indicate that Upf3b-deficient humans develop mental retardation, suggesting that even if Upf3a and Upf3b have overlapping functions in mammalian development, the loss of one of these paralogues is enough to perturb human development [39].

So far, only the core NMD factors (Upf1-Upf3) and Smg7 have been identified in plants. Mutants for Arabidopsis Upf1 are lethal and show metabolic, flowering, seeding and growth problems [40-42], and mutations in the Upf3 gene show strikingly similar phenotypes to the Upf1 mutants [40]. Smg7 mutants exhibit growth defects and infertility. In the case of the 
male, the germ cells fail to transit from anaphase to telophase in the second division, suggesting that Smg7 is required for exiting meiosis in Arabidopsis [43].

\section{Comparative analysis: why are some NMD effectors essential in some organisms, but not in others?}

The work done in fruitflies and vertebrates suggests that the loss of NMD causes lethality, whereas mutations in the main NMD effectors (Upf and Smg proteins) in yeast and nematode worms have no major developmental defects, indicating that the phenotype of NMD mutants seems to correlate with the complexity of the organism. Is this differential requirement for the NMD factors across species due to variability in the developmental importance of the RNA targets regulated by NMD, or instead due to additional essential functions that the NMD factors might have acquired in fruitflies and vertebrates?

For example, mammalian Upf1 is involved in Staufen-dependent mRNA decay, genome stability, histone mRNA degradation and telomere metabolism (reviewed in [6]), and human Upf1 also modulates the siRNA (short interfering RNA) pathway [44]. All of these Upf1 functions, except for telomere metabolism, have been shown to be independent of Upf2. In addition, Smg1 is involved in several stress responses in C. elegans and human cells, and Smg6 functions in telomere regulation [45-47]. These observations suggest that the lethal phenotype associated with some of the NMD mutants could result from blocking these functions. Supporting this hypothesis is the finding that in contrast with Upf1 and Upf2, Upf3 is not essential for cellular growth or animal viability in Drosophila (P. Avery, M. Vicente-Crespo and I.M. Palacios, unpublished work) or in fish [35]. This, in turn, implies that NMD might not be essential for development, and that the phenotypic lethality of the $U P F 1$ and UPF2 strong alleles might be due to their activity in other developmentally essential processes, rather than because of a loss of NMD. This hypothesis is supported further by the analysis of the Drosophila upf $2^{25 \mathrm{G}}$ mutant allele. This allele is thought to be completely compromised for NMD, yet, unlike upf2-null alleles, occasional upf $2^{25 \mathrm{G}}$ mutant males survive to adulthood [33].

Alternatively, the severity of the phenotype could be explained by an increase in the physiological role played by the transcripts regulated by NMD and/or by the increase in the number of PTC-containing isoforms. For example, the prevalence of alternative splicing (and therefore of PTC+ RNAs generated by alternative splicing) also increases in complex organisms [48]. In favour of this hypothesis is the fact that yeast Upf1 is also required for other processes in addition to NMD, such as telomere stability and protein degradation [49,50], but UPF1 is not an essential gene in Saccharomyces. In addition, it is not yet clear whether the role of the NMD factors in other cellular processes is always independent of their function in NMD. In particular, the telomere defects found in upf 1 mutant yeast are also shared by UPF2- and UPF3-deleted strains, suggesting that the NMD pathway regulates specific mRNAs that are important for telomere functions [49]. Further supporting this model is our finding that Drosophila Upf3 seems to have a peripheral role in the degradation of various transcripts that are strongly regulated by Upf 1 and Upf2, and that the allele upf $2^{25 \mathrm{G}}$, which was previously described as an NMD-null mutant, has weaker effects on NMD targets than other UPF2 alleles that are lethal (P. Avery, M. Vicente-Crespo and I.M. Palacios, unpublished work). Finally, we find that the interaction of Upf2 with Upf3 is not essential for fruitfly viability, whereas the binding of Upf2 to Upf1 is. This result suggests that the UPF1 and UPF2 lethality phenotype is probably due to a function of these proteins that requires the formation of the Upf1-Upf2 complex, as is the case for NMD.

All of these data show that there is a correlation between NMD target levels and lethality phenotype in the mutant alleles of upf1, upf 2 and upf3, suggesting that the differential effect 
on targets could explain why $U P F 1$ and $U P F 2$, but not $U P F 3$, are essential genes in the fruitfly. If we were to apply these arguments to the observations across species, it is possible that the differential effects on, and/or the nature of, targets could also explain why some NMD factors are essential genes in some organisms, but not in others.

\section{Concluding remarks}

Although the identification of an individual bona fide NMD transcript may in turn suggest a specific functional role for NMD, it is important that this be validated with the analysis of phenotype, especially when considering that the secondary effects of depleting NMD could go further than the mere stabilization of the direct targets. NMD targets that are responsible for any of the mutant developmental phenotypes described so far have not yet been identified. A more comprehensive analysis of the NMD targets throughout development should give us a better picture of which targets need to be under strict control of the NMD pathway to ensure the organism's survival.

\section{Acknowledgments}

We thank Katrina Gold and Paul Avery for sharing unpublished observations, and Lucy S. Williams for comments.

Funding

M.V.-C. is supported by the National Institutes of Health. I.M.P. is a Royal Society University Research Fellow.

\section{Abbreviations used}

$\begin{array}{ll}\text { EJC } & \text { exon junction complex } \\ \text { NMD } & \text { nonsense-mediated mRNA decay } \\ \text { PTC } & \text { premature termination codon } \\ \text { Smg } & \text { suppressor with morphological effect on genitalia } \\ \text { Upf } & \text { up-frameshift suppressor }\end{array}$

\section{References}

1. Alonso CR. Nonsense-mediated RNA decay: a molecular system micromanaging individual gene activities and suppressing genomic noise. BioEssays. 2005; 27:463-466. [PubMed: 15832387]

2. Gudikote JP, Wilkinson MF. T-cell receptor sequences that elicit strong down-regulation of premature termination codon-bearing transcripts. EMBO J. 2002; 21:125-134. [PubMed: 11782432]

3. Mitrovich QM, Anderson P. Unproductively spliced ribosomal protein mRNAs are natural targets of mRNA surveillance in C. elegans. Genes Dev. 2000; 14:2173-2184. [PubMed: 10970881]

4. He F, Peltz SW, Donahue JL, Rosbash M, Jacobson A. Stabilization and ribosome association of unspliced pre-mRNAs in a yeast upf1 ${ }^{-}$mutant. Proc. Natl. Acad. Sci. U.S.A. 1993; 90:7034-7038. [PubMed: 8346213]

5. Neu-Yilik G, Gehring NH, Hentze MW, Kulozik AE. Nonsense-mediated mRNA decay: from vacuum cleaner to Swiss army knife. Genome Biol. 2004; 5:218. [PubMed: 15059251]

6. Nicholson P, Yepiskoposyan H, Metze S, Zamudio Orozco R, Kleinschmidt N, Muhlemann O. Nonsense-mediated mRNA decay in human cells: mechanistic insights, functions beyond quality control and the double-life of NMD factors. Cell. Mol. Life Sci. 2010; 67:677-700. [PubMed: 19859661]

7. Lewis BP, Green RE, Brenner SE. Evidence for the widespread coupling of alternative splicing and nonsense-mediated mRNA decay in humans. Proc. Natl. Acad. Sci. U.S.A. 2003; 100:189-192. [PubMed: 12502788] 
8. Green RE, Lewis BP, Hillman RT, Blanchette M, Lareau LF, Garnett AT, Rio DC, Brenner SE. Widespread predicted nonsense-mediated mRNA decay of alternatively-spliced transcripts of human normal and disease genes. Bioinformatics. 2003; 19(Suppl. 1):I118-I121. [PubMed: 12855447]

9. Hillman RT, Green RE, Brenner SE. An unappreciated role for RNA surveillance. Genome Biol. 2004; 5:R8. [PubMed: 14759258]

10. Culbertson MR. RNA surveillance Unforeseen consequences for gene expression, inherited genetic disorders and cancer. Trends Genet. 1999; 15:74-80. [PubMed: 10098411]

11. Vincent MC, Pujo AL, Olivier D, Calvas P. Screening for PAX6 gene mutations is consistent with haploin sufficiency as the main mechanism leading to various ocular defects. Eur. J. Hum. Genet. 2003; 11:163-169. [PubMed: 12634864]

12. Hutchinson S, Furger A, Halliday D, Judge DP, Jefferson A, Dietz HC, Firth H, Handford PA. Allelic variation in normal human FBN1 expression in a family with Marfan syndrome: a potential modifier of phenotype? Hum. Mol. Genet. 2003; 12:2269-2276. [PubMed: 12915484]

13. Wilkinson MF. A new function for nonsense-mediated mRNA-decay factors. Trends Genet. 2005; 21:143-148. [PubMed: 15734573]

14. Holbrook JA, Neu-Yilik G, Hentze MW, Kulozik AE. Nonsense-mediated decay approaches the clinic. Nat. Genet. 2004; 36:801-808. [PubMed: 15284851]

15. Pastor F, Kolonias D, Giangrande PH, Gilboa E. Induction of tumour immunity by targeted inhibition of nonsense-mediated mRNA decay. Nature. 2010; 465:227-230. [PubMed: 20463739]

16. Chamieh H, Ballut L, Bonneau F, Le Hir H. NMD factors UPF2 and UPF3 bridge UPF1 to the exon junction complex and stimulate its RNA helicase activity. Nat. Struct. Mol. Biol. 2008; 15:85-93. [PubMed: 18066079]

17. Isken O, Kim YK, Hosoda N, Mayeur GL, Hershey JW, Maquat LE. Upf1 phosphorylation triggers translational repression during nonsense-mediated mRNA decay. Cell. 2008; 133:314327. [PubMed: 18423202]

18. Ohnishi T, Yamashita A, Kashima I, Schell T, Anders KR, Grimson A, Hachiya T, Hentze MW, Anderson P, Ohno S. Phosphorylation of hUPF1 induces formation of mRNA surveillance complexes containing hSMG-5 and hSMG-7. Mol. Cell. 2003; 12:1187-1200. [PubMed: 14636577]

19. Rehwinkel J, Raes J, Izaurralde E. Nonsense-mediated mRNA decay: target genes and functional diversification of effectors. Trends Biochem. Sci. 2006; 31:639-646. [PubMed: 17010613]

20. Muramatsu R, Morita T, Iwamatsu A, Hachiya T, et al. SMG-8 and SMG-9, two novel subunits of the SMG-1 complex, regulate remodeling of the mRNA surveillance complex during nonsensemediated mRNA decay. Genes Dev. 2009; 23:1091-1105. [PubMed: 19417104]

21. Gatfield D, Izaurralde E. Nonsense-mediated messenger RNA decay is initiated by endonucleolytic cleavage in Drosophila. Nature. 2004; 429:575-578. [PubMed: 15175755]

22. Huntzinger E, Kashima I, Fauser M, Sauliere J, Izaurralde E. SMG6 is the catalytic endonuclease that cleaves mRNAs containing nonsense codons in metazoan. RNA. 2008; 14:2609-2617. [PubMed: 18974281]

23. Eberle AB, Lykke-Andersen S, Muhlemann O, Jensen TH. SMG6 promotes endonucleolytic cleavage of nonsense mRNA in human cells. Nat. Struct. Mol. Biol. 2009; 16:49-55. [PubMed: 19060897]

24. He F, Jacobson A. Identification of a novel component of the nonsense-mediated mRNA decay pathway by use of an interacting protein screen. Genes Dev. 1995; 9:437-454. [PubMed: 7883168]

25. Leeds P, Peltz SW, Jacobson A, Culbertson MR. The product of the yeast UPF1 gene is required for rapid turnover of mRNAs containing a premature translational termination codon. Genes Dev. 1991; 5:2303-2314. [PubMed: 1748286]

26. Cui Y, Hagan KW, Zhang S, Peltz SW. Identification and characterization of genes that are required for the accelerated degradation of mRNAs containing a premature translational termination codon. Genes Dev. 1995; 9:423-436. [PubMed: 7883167]

27. Lee BS, Culbertson MR. Identification of an additional gene required for eukaryotic nonsense mRNA turnover. Proc. Natl. Acad. Sci. U.S.A. 1995; 92:10354-10358. [PubMed: 7479783] 
28. Mendell JT, Medghalchi SM, Lake RG, Noensie EN, Dietz HC. Novel Upf2p orthologues suggest a functional link between translation initiation and nonsense surveillance complexes. Mol. Cell. Biol. 2000; 20:8944-8957. [PubMed: 11073994]

29. Wen J, Brogna S. Splicing-dependent NMD does not require the EJC in Schizosaccharomyces pombe. EMBO J. 2010; 29:1537-1551. [PubMed: 20360683]

30. Longman D, Plasterk RH, Johnstone IL, Cáceres JF. Mechanistic insights and identification of two novel factors in the $C$. elegans NMD pathway. Genes Dev. 2007; 21:1075-1085. [PubMed: 17437990]

31. Hodgkin J, Papp A, Pulak R, Ambros V, Anderson P. A new kind of informational suppression in the nematode Caenorhabditis elegans. Genetics. 1989; 123:301-313. [PubMed: 2583479]

32. Rehwinkel J, Letunic I, Raes J, Bork P, Izaurralde E. Nonsense-mediated mRNA decay factors act in concert to regulate common mRNA targets. RNA. 2005; 11:1530-1544. [PubMed: 16199763]

33. Metzstein MM, Krasnow MA. Functions of the nonsense-mediated mRNA decay pathway in Drosophila development. PLoS Genet. 2006; 2:e180. [PubMed: 17196039]

34. Palacios IM, Gatfield D, St Johnston D, Izaurralde E. An eIF4AIII-containing complex required for mRNA localization and nonsense-mediated mRNA decay. Nature. 2004; 427:753-757. [PubMed: 14973490]

35. Wittkopp N, Huntzinger E, Weiler C, Sauliere J, Schmidt S, Sonawane M, Izaurralde E. Nonsensemediated mRNA decay effectors are essential for zebrafish embryonic development and survival. Mol. Cell. Biol. 2009; 29:3517-3528. [PubMed: 19414594]

36. Medghalchi SM, Frischmeyer PA, Mendell JT, Kelly AG, Lawler AM, Dietz HC. Rent1, a transeffector of nonsense-mediated mRNA decay, is essential for mammalian embryonic viability. Hum. Mol. Genet. 2001; 10:99-105. [PubMed: 11152657]

37. Weischenfeldt J, Damgaard I, Bryder D, Theilgaard-Monch K, Thoren LA, Nielsen FC, Jacobsen SE, Nerlov C, Porse BT. NMD is essential for hematopoietic stem and progenitor cells and for eliminating by-products of programmed DNA rearrangements. Genes Dev. 2008; 22:1381-1396. [PubMed: 18483223]

38. Azzalin CM, Lingner J. The human RNA surveillance factor UPF1 is required for S phase progression and genome stability. Curr. Biol. 2006; 16:433-439. [PubMed: 16488880]

39. Tarpey PS, Raymond FL, Nguyen LS, Rodriguez J, Hackett A, Vandeleur L, Smith R, Shoubridge C, Edkins S, Stevens C, et al. Mutations in UPF3B, a member of the nonsense-mediated mRNA decay complex, cause syndromic and nonsyndromic mental retardation. Nat. Genet. 2007; 39:1127-1133. [PubMed: 17704778]

40. Arciga-Reyes L, Wootton L, Kieffer M, Davies B. UPF1 is required for nonsense-mediated mRNA decay (NMD) and RNAi in Arabidopsis. Plant. J. 2006; 47:480-489. [PubMed: 16813578]

41. Yoine M, Ohto MA, Onai K, Mita S, Nakamura K. The lba1 mutation of UPF1 RNA helicase involved in nonsense-mediated mRNA decay causes pleiotropic phenotypic changes and altered sugar signalling in Arabidopsis. Plant J. 2006; 47:49-62. [PubMed: 16740149]

42. Yoine M, Nishii T, Nakamura K. Arabidopsis UPF1 RNA helicase for nonsense-mediated mRNA decay is involved in seed size control and is essential for growth. Plant. Cell Physiol. 2006; 47:572-580. [PubMed: 16540482]

43. Kerenyi Z, Merai Z, Hiripi L, Benkovics A, Gyula P, Lacomme C, Barta E, Nagy F, Silhavy D. Inter-kingdom conservation of mechanism of nonsense-mediated mRNA decay. EMBO J. 2008; 27:1585-1595. [PubMed: 18451801]

44. Jin H, Suh MR, Han J, Yeom KH, Lee Y, Heo I, Ha M, Hyun S, Kim VN. Human UPF1 participates in small RNA-induced mRNA downregulation. Mol. Cell. Biol. 2009; 29:5789-5799. [PubMed: 19704008]

45. Brumbaugh KM, Otterness DM, Geisen C, Oliveira V, Brognard J, Li X, Lejeune F, Tibbetts RS, Maquat LE, Abraham RT. The mRNA surveillance protein hSMG-1 functions in genotoxic stress response pathways in mammalian cells. Mol. Cell. 2004; 14:585-598. [PubMed: 15175154]

46. Masse I, Molin L, Mouchiroud L, Vanhems P, Palladino F, Billaud M, Solari F. A novel role for the SMG-1 kinase in lifespan and oxidative stress resistance in Caenorhabditis elegans. PLoS ONE. 2008; 3:e3354. [PubMed: 18836529] 
47. Chawla R, Azzalin CM. The telomeric transcriptome and SMG proteins at the crossroads. Cytogenet. Genome Res. 2008; 122:194-201. [PubMed: 19188687]

48. Kim E, Magen A, Ast G. Different levels of alternative splicing among eukaryotes. Nucleic Acids Res. 2007; 35:125-131. [PubMed: 17158149]

49. Lew JE, Enomoto S, Berman J. Telomere length regulation and telomeric chromatin require the nonsense-mediated mRNA decay pathway. Mol. Cell. Biol. 1998; 18:6121-6130. [PubMed: 9742129]

50. Kuroha K, Tatematsu T, Inada T. Upf1 stimulates degradation of the product derived from aberrant messenger RNA containing a specific nonsense mutation by the proteasome. EMBO Rep. 2009; 10:1265-1271. [PubMed: 19798102] 
A

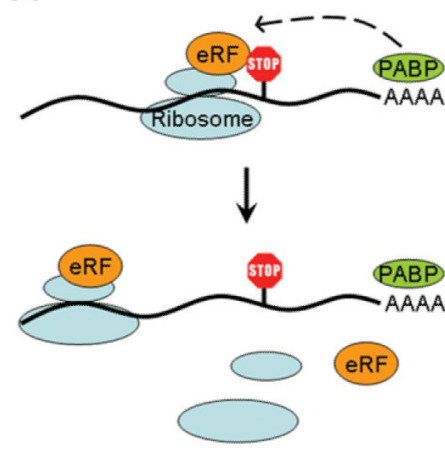

B

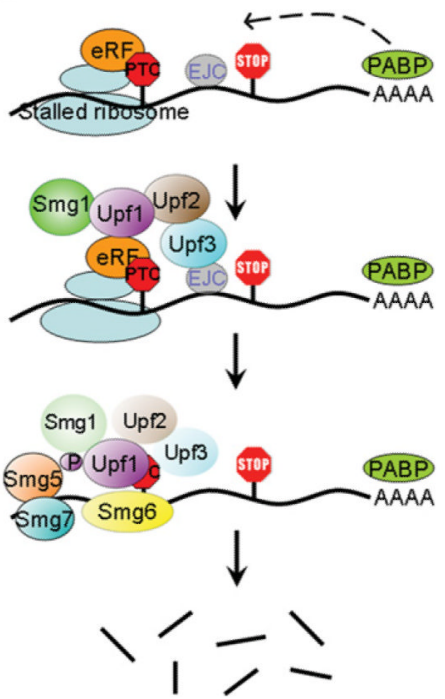

Figure 1. NMD pathway

(A) Simplified model for translation in the proper mRNP (messenger ribonucleoprotein) environment. In the case of mRNAs with a normal stop codon, signals from the $3^{\prime}$-UTR (untranslated region) and the poly(A) tail (RNA features and/or RNA-binding proteins) allow the proper termination of translation and reinitiation of the ribosome at the start codon. (B) Simplified model for assembly of the NMD machinery on an NMD target. When a stop codon fails to get the signals from the $3^{\prime}$-UTR and the poly(A) tail (PTC), the ribosome stalls and the NMD factor Upf1 is recruited by the termination factors. The kinase Smg1 is also incorporated and phosphorylates Upf1, committing the PTC-containing mRNA to NMD. Upf 2 and, to a lesser extent, Upf3 are necessary for Upf1 phosphorylation. In some cases, events such as the presence of the EJC downstream of the PTC enhance the recognition of the mRNA as an NMD target (e.g. by facilitating Upf2 and Upf3 recruitment). Phosphorylated Upf1 interacts with the other Smg factors, Smg5, Smg6 and Smg7, and mRNA degradation is initiated. eRF, eukaryotic translation release factor; PABP, poly(A)-binding protein. 


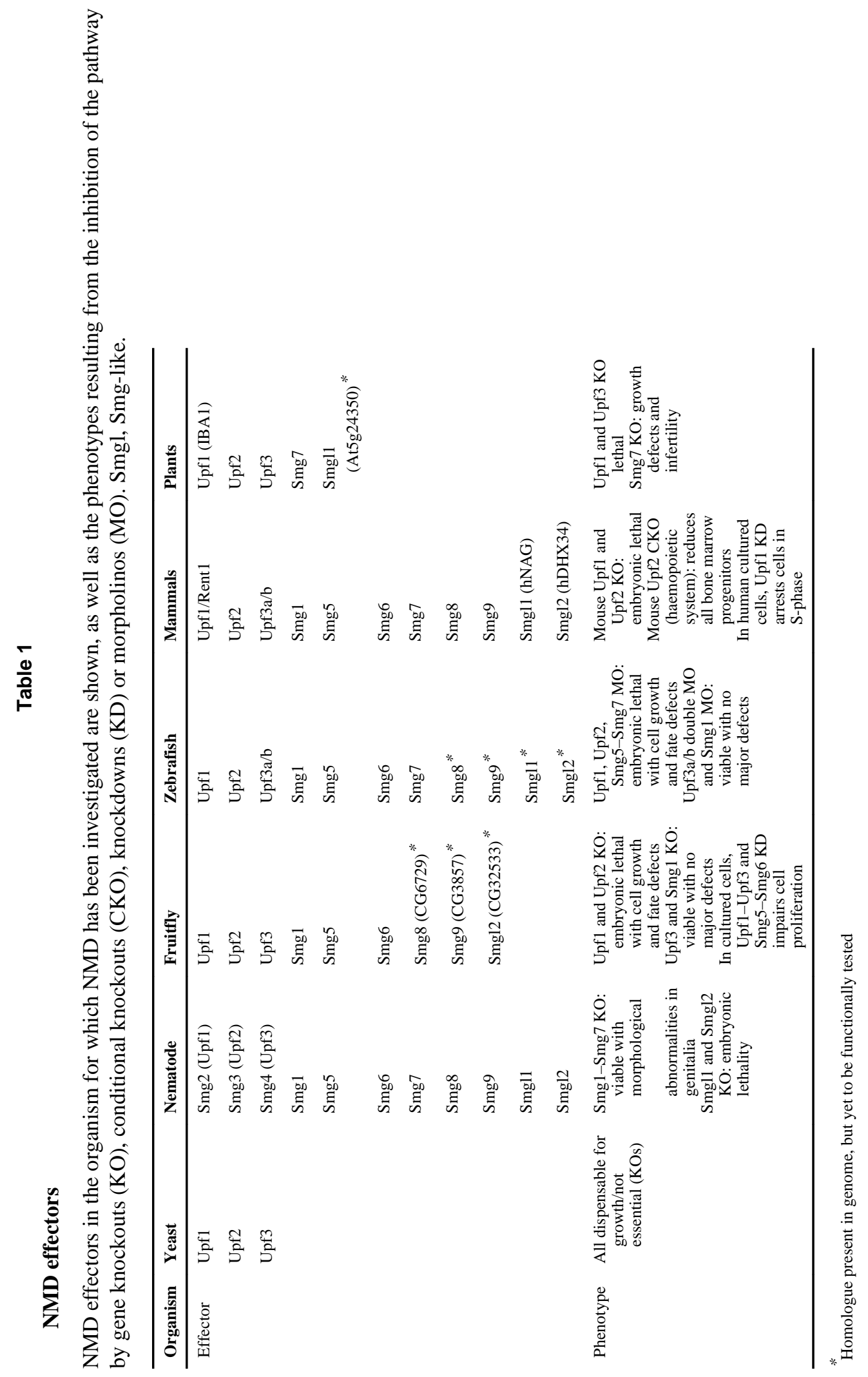

